\title{
Directivity of basic linear arrays
}

\section{Bach, Henning}

\section{Published in:}

I E E E Transactions on Antennas and Propagation

Publication date:

1970

\section{Document Version}

Publisher's PDF, also known as Version of record

Link back to DTU Orbit

\section{Citation (APA):}

Bach, H. (1970). Directivity of basic linear arrays. I E E E Transactions on Antennas and Propagation, 18(1), 107-110.

\section{General rights}

Copyright and moral rights for the publications made accessible in the public portal are retained by the authors and/or other copyright owners and it is a condition of accessing publications that users recognise and abide by the legal requirements associated with these rights.

- Users may download and print one copy of any publication from the public portal for the purpose of private study or research.

- You may not further distribute the material or use it for any profit-making activity or commercial gain

- You may freely distribute the URL identifying the publication in the public portal

If you believe that this document breaches copyright please contact us providing details, and we will remove access to the work immediately and investigate your claim 


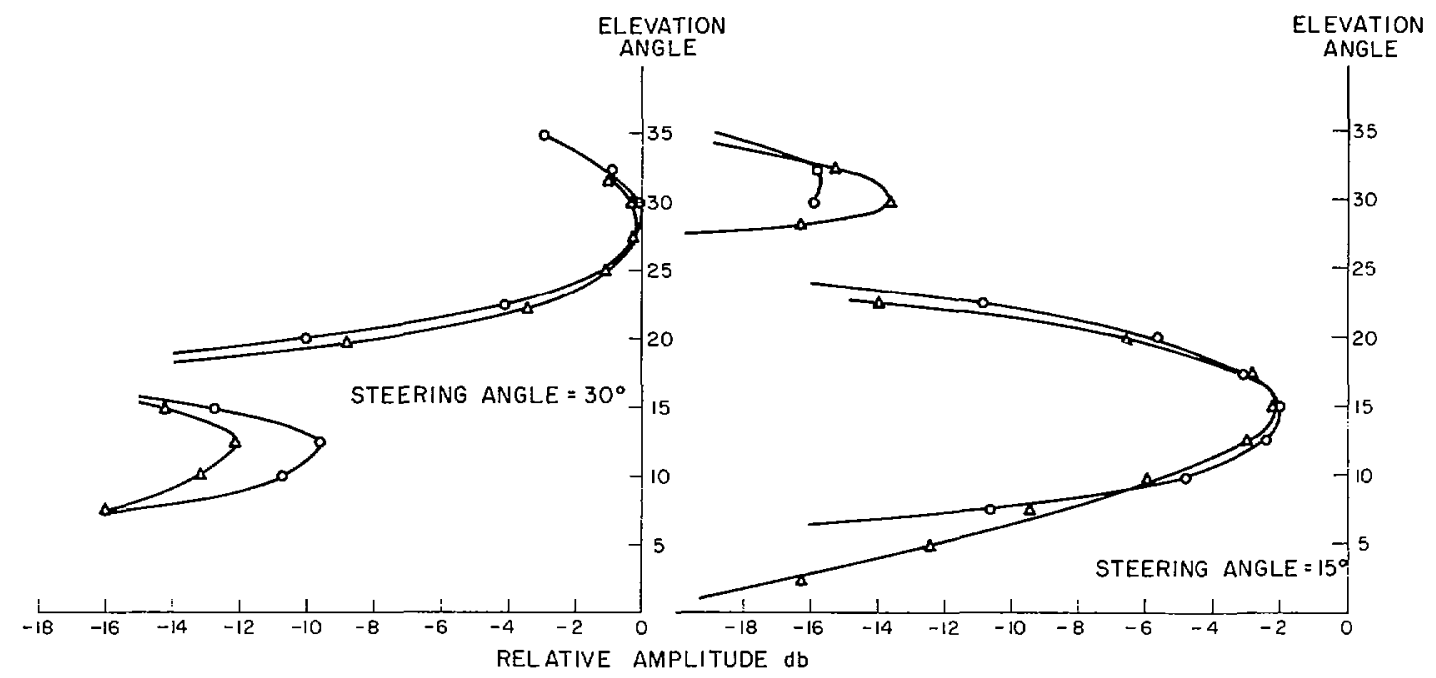

Fig. 4. Elevation pattern steering with cosine illumination five elements over screen. $O$ : infinite plane, perfect conditions. $\triangle: 16 \lambda$ screen on agriculturai soil.

where

$$
\begin{aligned}
\tau_{0 n} & =(2 \pi d / \lambda)\left[\frac{1}{2}+(n-1)\right] \sin \alpha_{0} \\
\alpha= & \text { look angle } \\
\alpha_{0}= & \text { steering angle } \\
d= & \text { element spacing; first element at } d / 2 \text { above ground } \\
& \text { plane }
\end{aligned}
$$

$W^{\prime \prime}(n, \alpha)=$ pattern of $n$th element above screen [eq. (2) ].

For a perfect ground of infinite extent,

$$
E\left(\alpha_{0}, \alpha\right)=\sum_{n=1}^{n=5} \cos \tau_{0 n} \cos \tau_{n}
$$

Figs. 3 and 4 show the results for several steering angles.

Note that the peak of the main beam points in the desired direction when the steering angle is greater than $10^{\circ}$, which is approximately one $3-\mathrm{dB}$ beamwidth of the effective aperture (actual aperture and images), but does not do so below that angle. However, except for $0^{\circ}$, a case of academic interest only, this limitation applies also to perfect ground of infinite extent (Section IV).

\section{Disclssion of Low Angle Steering Limitations}

Consider for simplicity an infinite plane of perfect conductivity. The cosine illumination (9) can be expressed as the sum

$$
\frac{1}{2}\left(\exp \left\{j\left[(2 \pi / \lambda) h_{i} \sin \alpha_{0}\right]\right\}+\exp \left\{-j\left[(2 \pi / \lambda) h_{i} \sin \alpha_{0}\right]\right\}\right) .
$$

This is equivalent to applying the sum of complex conjugate excitations to every element, one for steering to $+\alpha_{0}$, the other to $-\alpha_{0}$. For vertical polarization one can postulate the existence of image elements of the same excitation as the actual elements. The terms

$$
\exp \left\{-j\left[(2 \pi / \lambda) h_{i} \sin \alpha_{0}\right]\right\}
$$

of the elements and

$$
\exp \left\{+j\left[(2 \pi / \lambda) h_{i} \sin \alpha_{0}\right]\right\}
$$

of the images form precisely the right phase front for steering to $+\alpha_{0}$; similarly, the other conjugate sets form a phase front for steering to $-\alpha_{0}$. We thus have in effect two patterns, each corresponding to twice the aperture, steered in opposite direction with the restriction, of course, that only that part of the downward steered pattern exists which lies above the earth. At zero degrees, the two patterns coalesce into one, but at all other angles there are various degrees of interference. Within elevation angles of one 3-dB beamwidth, the interference is sufficiently strong to caluse beam distortions, resulting in significant departures from the desired pointing direction. Increasing the aperture, therefore, results in an increase in the potential steering region.
The situation is more complicated in the case of inperfect ground, which includes the finite ground screen on any soil. Two rotaing patterns can again be postulated but they are different from those in the preceeding case and are complex (1ather than real). The interference results in an undercut pattem below the pseudoBrewster angle and essentially the same results as before above it. Consequently, to extend steering to lower angles requires both a more nearly perfect ground plane and a higher aperture. The practical way to improve the ground plane is to lengthen the screen.

\section{Coxclusions}

The analysis demonstrated the utility of the sinusoidal illumination for elevation steering of the pattern of an array over a modest ground screen ( 16 wavelengths $=480$ meters at $10 \mathrm{MH} \%$ ). For a screen and an aperture of about 16 and 2 wavelengths, respectively, the effective steering region starts at about $10^{\circ}$. The steering region can be extended to lower angles by increasing both the screen and the aperture.

It is reasonable to expect that simple approximations to a sinusoidal illumination, for example a square wave $( \pm 1)$, can be used to steer the beam if the attendant high secondary lobes, due to harmonies are acceptable.

\section{ACKNOWLEDGMENT}

Very valuable contributions by $\mathrm{S}$. $\mathrm{N}$. Hunt of the Mitre Corporation are gratefully acknowledged.

\section{References}

ABRAHAM WALDMAT The Mitre Corp. Bedford, Mass. 01730

[1] J. R. Wait, "On the theory of radiation from a raised electric dipole over an inhomogeneous ground plane," Radio Sci., vol. 2, September

[2] R. G. FitzGerell, A. O. Wilson, L. I. Proctor, and H. Y. Cottony, "An HF array antenna electronically scanned in elevation," IEEE Trans. Antennas and Propagation, vol. AP-15, pp. 758-766, Nơember 1967 .

\section{Directivity of Basic Linear Arrays}

Abstract-For a linear uniform array of $n$ elements, an expression is derived for the directivity as a function of the spacing and the phase constants. The cases of isotropic elements, collinear short

Manuscript received March 17, 1969; revised June 12, 1969. 
dipoles, and parallel short dipoles are included. The formula obtained is discussed in some detail and contour diagrams of the directivity as a function of the spacing and the phase constants in the above-mentioned cases are exhibited.

\section{INTRODUCTION}

Among the large variety of arrays of radiating elements, the simplest type is the uniform linear array. This array is completely specified by the spacing and the phase progression constants. In what follows, the directive properties of uniform arrays of isotropic sources, parallel dipoles, and collinear dipoles are considered as a funetion of the spacing and phase constants.

\section{Derivation of the Directivity}

The directivity $D(\theta, \phi)$ of any array is defined by

$$
D(\theta, \phi)=P(\theta, \phi) / I, \quad I=(1 / 4 \pi) \int_{4 \pi} P(\theta, \phi) d \Omega
$$

where $P(\theta, \phi)$ is the power pattern and $d \Omega$ an element of solid angle. For a linear uniform array with the elements located on the $z$ axis $(\theta=0)$ of a rectangular coordinate system, the power pattern is given by

$$
P(\theta, \phi)=f^{2}(\theta, \phi) g^{2}(\theta)
$$

where $f(\theta, \phi)$ is the radiation pattern of the individual element and $g(\theta)$ the array factor. When we denote the spacing between consecutive elements by $d$ and the phase progression constant by $\delta$, the array factor

$$
g(\theta)=\left|\frac{\sin n(\gamma / 2)}{n \sin (\gamma / 2)}\right|, \quad \gamma=\delta+k d \cos \theta
$$

where $n$ is the number of elements. Now

$$
\begin{aligned}
f^{2}(\theta, \phi) & =1 & & \text { for isotropic elements } \\
& =\sin ^{2} \theta & & \text { for collinear short dipoles } \\
& =1-\sin ^{2} \theta \cos ^{2} \phi & & \text { for parallel short dipoles }
\end{aligned}
$$

and it is easily shown that the integral (1) in the three cases may be written

$$
I_{\text {iso }}=I_{1}, \quad I_{\text {col }}=I_{1}-I_{2,}, \quad I_{\mathrm{par}}=\frac{1}{2}\left(I_{1}+I_{2}\right)
$$

where

$$
\begin{aligned}
& I_{1}=\frac{1}{4 \pi} \int_{4 \pi}\left[\frac{\sin n(\gamma / 2)}{n \sin (\gamma / 2)}\right]^{2} d \Omega \\
& I_{2}=\frac{1}{4 \pi} \int_{4 \pi} \cos ^{2} \theta\left[\frac{\sin n(\gamma / 2)}{n \sin (\gamma / 2)}\right]^{2} d \Omega .
\end{aligned}
$$
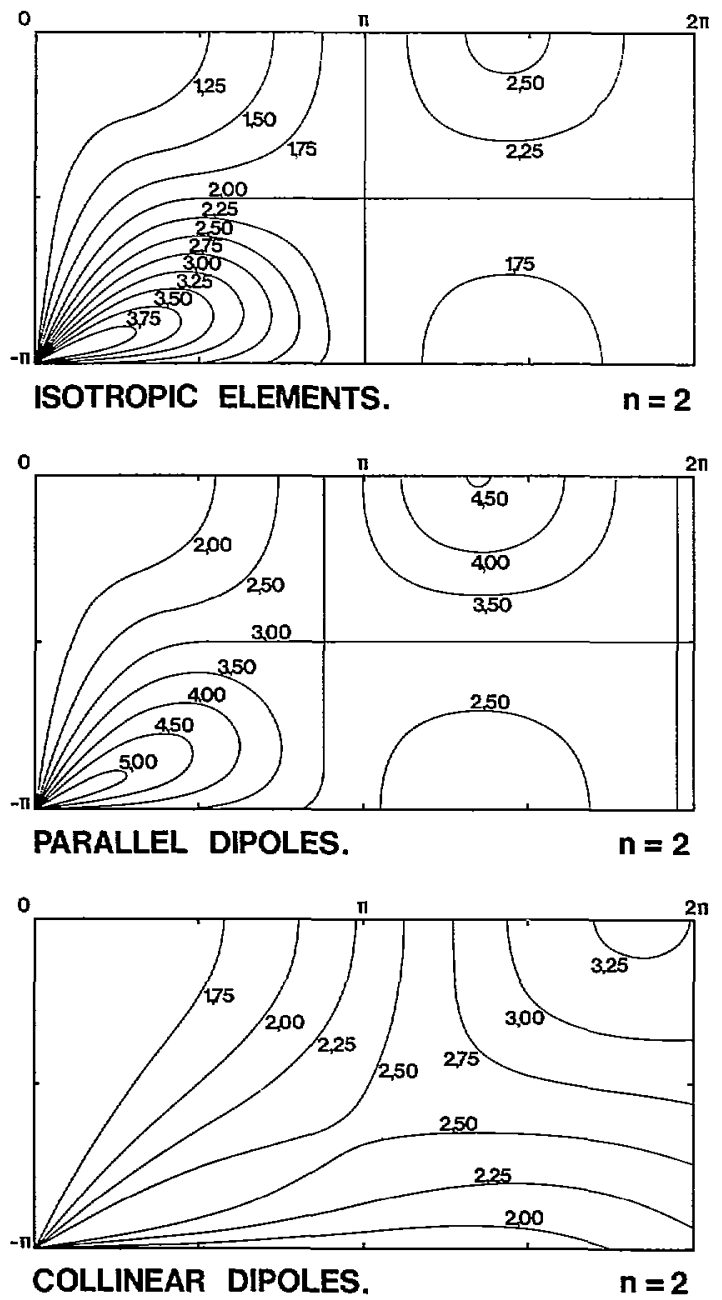

Fig. 1.

and

$$
\begin{aligned}
I_{2}=\frac{1}{3 n}+ & \frac{2}{n^{2}} \sum_{m=1}^{n-1}\left\{\frac{(n-m)}{m k d}\right. \\
& \left.\cdot\left[\left(1-2 / m^{2} k^{2} d^{2}\right) \sin m k d+(2 / m k d) \cos m k d\right] \cos m \delta\right\}
\end{aligned}
$$

may be found by direct integration. The final result may be summarized as follows:

$$
D(\theta, \phi)=f^{2}(\theta, \phi) g^{2}(\theta) /\left\{a_{0} / n+\left(2 / n^{2}\right) \sum_{m=1}^{n-1}[(n-m) / m k d]\left(a_{1} \sin m k d+a_{2} \cos m k d\right) \cos m \delta\right\}
$$

By introducing $d \Omega=2 \pi \sin \theta d \theta$ and using the finite series [1]

$$
\left[\frac{\sin n(\gamma / 2)}{n \sin (\gamma / 2)}\right]^{2}=\frac{1}{n}+\frac{2}{n^{2}} \sum_{m=1}^{n-1}(n-m) \cos m \gamma
$$

the results

$$
I_{1}=\frac{1}{n}+\frac{2}{n^{2}} \sum_{m=1}^{n-1} \frac{n-m}{m k d} \sin m k d \cos m \delta
$$

where $f^{2}(\theta, \phi), a_{0}, a_{1}$, and $a_{2}$ are given in Table I.

The above formula has been derived in [2] using the same method, but in a less general and slightly modified form. For $\delta=0$ and $\delta=-k d$, (3) reduces to the formulas derived by Tai [3] by means of the mutual impedance concept.

\section{Discussiox}

In Figs. 1-3 are shown contour diagrams of the maximum directivity of arrays of 2,3 , and 6 elements as a function of the spacing constant $k d$ and the phase constant $\delta$. Further diagrams 

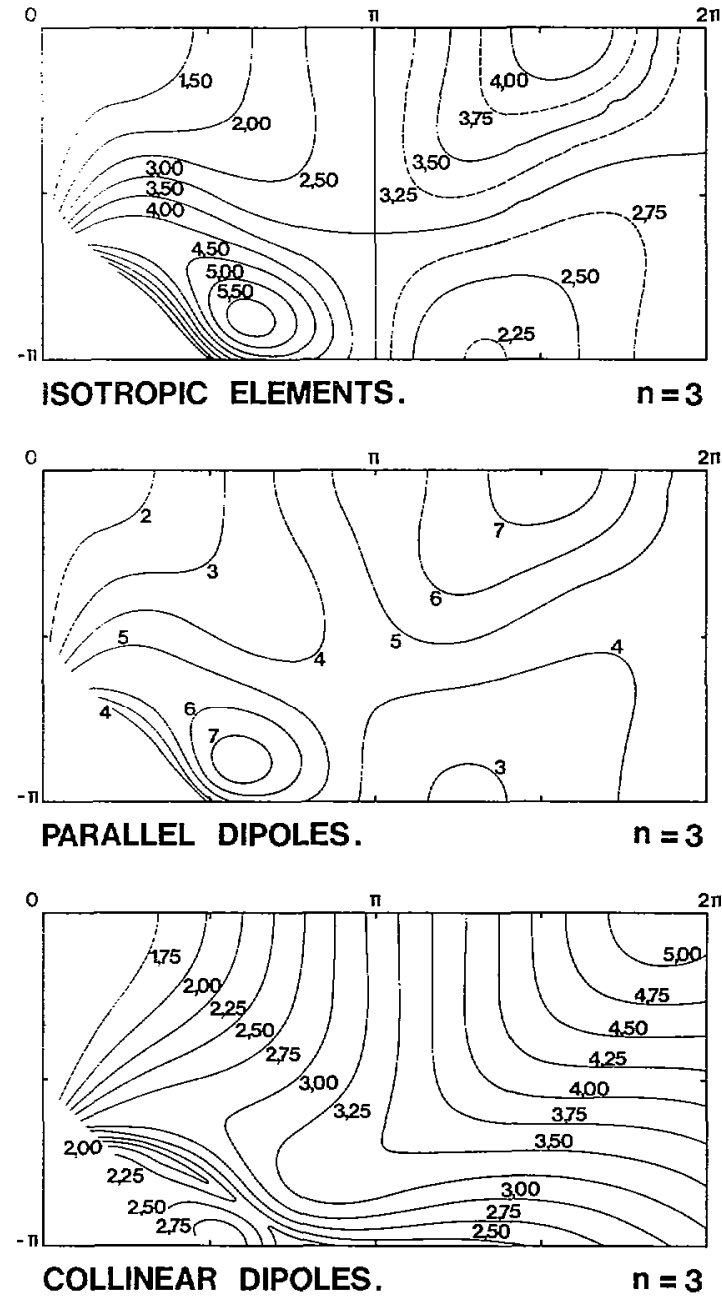

Fig. 2.

TABLE I

\begin{tabular}{lcccc}
\hline \hline & $f^{2}(\theta, \phi)$ & $a_{0}$ & $a_{1}$ & $a_{2}$ \\
\hline Isotropic elements & 1 & 1 & 1 & 0 \\
Collinear dipoles & $\sin ^{2} \theta$ & $\frac{2}{3}$ & $\frac{2}{(m k d)^{2}}$ & $-\frac{2}{m k d}$ \\
& & & & \\
Parallel dipoles & $1-\sin ^{2} \theta \cos ^{2} \phi$ & $\frac{2}{3}$ & $1-\frac{1}{(m k d)^{2}}$ & $\frac{1}{m k d}$
\end{tabular}

are presented in [4]. The line $\delta=0$ corresponds to broadside arrays and the line $\delta=-k d$ to ordinary endfire arrays, while the domain between these lines corresponds to arrays with other or additional maximum directions. In the area between $\delta=-k d$ and $\delta=-\pi$, the array is still endfire but the magnitude of the main lobe is reduced compared to the magnitude obtained when the condition for ordinary endfire radiation is fulfilled. For $n \geq 3$ the magnitude of the main lobe may become less than the largest sidelobe. The part of each diagram corresponding to such values of $\delta$ and $k d$ is left empty.

It is seen from (3) that for $k d=p \pi, p=1,2,3, \cdots$, the maximum directivity is independent of $\delta$ and equal to the number of elements $n$. For a broadside array $(\delta=0), D_{\text {iso }}=n$ when $k d=p \pi$ while for an endfire array this value is obtained when $k d=p \pi / 2$. From
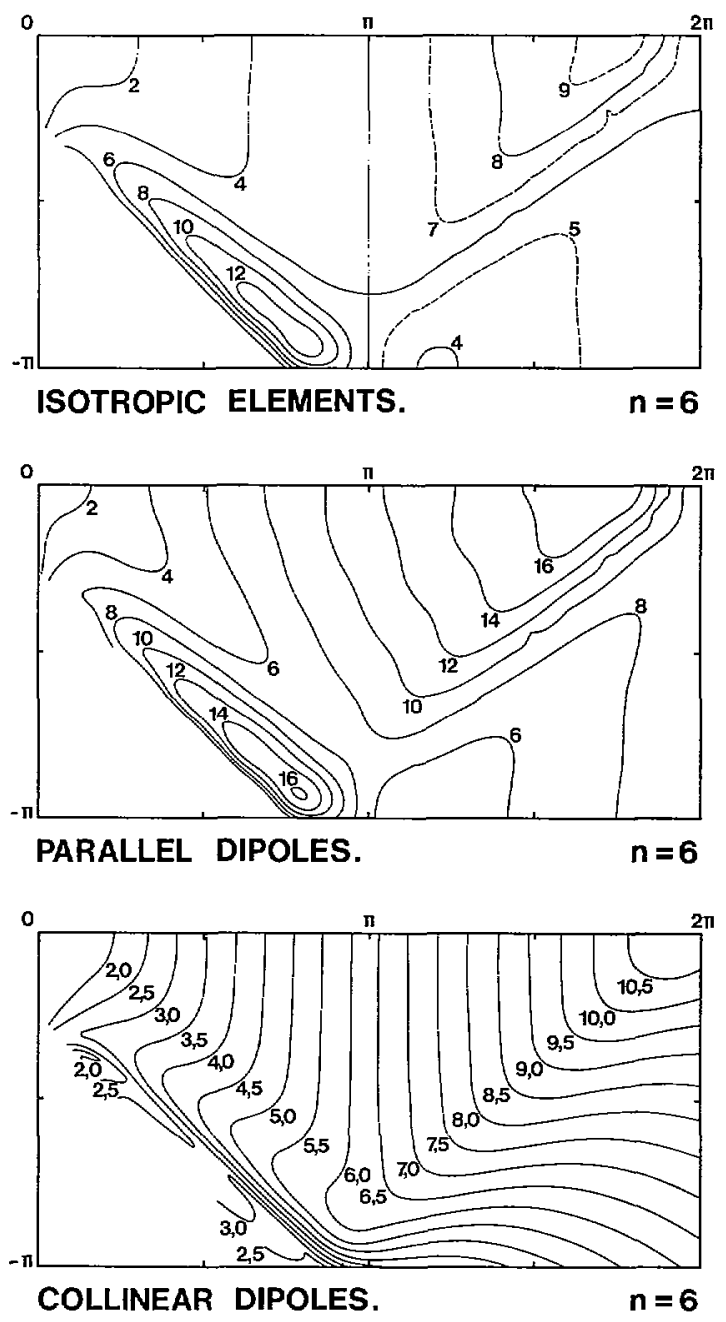

Fig. 3.

the contour diagrams (for $n=3$ and 6 ) it appears that in addition a wavy contour $D_{\text {iso }}=n$ exists and that the directivity surface exhibits saddle points on the lines $k d=p_{\pi}$. Two absolute maxima are present in the figures. The broadside maximum is obtained when $k d$ is somewhat less than $2 \pi$, and it is noted that the directivity decreases sharply beyond this maximum due to the appearance of grating lobes in the radiation pattern. The endfire maximum is obtained when $k d$ is somewhat less than $\pi$. This maximum is not obtained with an ordinary endfire array $\delta=-k d$ and it is in this context interesting to consider the Hansen-Woodyard condition for increased directivity of endfire arrays [5]. These authors found that an increased directivity could be obtained, if, for an ordinary endfire array, the phase constant was changed to

$$
\delta=-k d-\pi /(n-1)
$$

where $n$ is the number of elements. The above expression may be depicted as a straight line in the present diagrams. It is seen that it yields larger directivity than the corresponding ordinary endfire condition, but also that even higher directivity may be obtained by a uniform endfire array.

The diagram for $n=2$ (Fig. 1) is of special interest because the point $(k d, \delta)=(0,-\pi / 2)$ is included. It is well known that the theoretical maximum directivity of an equispaced linear array is equal to $n^{2}$ [6], but this optimum value is in general not obtained for an array with uniform excitation. However, in the case of $n=2$, the optimum array is also a uniform array and the optimum value $D_{\text {iso }}{ }^{0}=4$ may be obtained from (3) for $k d \rightarrow 0$ and $\delta \rightarrow-180$. 
The tricky nature of this optimum is illustrated by the fact that it is obtained only when the constraint $\delta=\frac{1}{3} k d-\pi$ is introduced, and the contour diagram illustrates the complicated structure of the directivity surface in the vicinity of this point.

The center diagrams in each figure apply for arrays of parallel dipoles. The structure of the diagrams is similar to that for isotropic elements, but some distortion results, especially in the upper parts of the diagrams. It should be noted that the position of the broadside and endfire maxima is almost unchanged when the isotropic elements are replaced by parallel dipoles.

The bottom diagrams apply for collinear dipoles. In this case, no endfire radiation is obtained and a marked distortion of the diagrams results. Very low directivities are obtained in the endfire area and, furthermore, the broadside maximum is shifted in the direction of $k d=2 \pi$.

\section{HeNning Bach}

Lab. of Electromagnetic Theory Technical University of Denmark Lyngby, Denmark

\section{REFERENCES}

[1] E. T. Whittaker and G. N. Watson, A Course of Modern Analysis.

[2] H. Zuhrt, Elektromagnetische Strahlungsfelder. Berlin: Springer,

[3] C. T. T. Tai " The nominal directivity of uniformly spaced arrays of dipoles," Microwave J., vol. 7, pp. 51-55, September" 1964 .

[4] H. Bach, "Directivity of basic linear arrays," Lab. of Electro-

magnetic Theory, Technical University of Denmark, $R$. 68 .

[5] W. W. Hansen and J. R. Woodyard, "A new principle in directional antenna design," Proc. IRE, vol. 26 , pp. $333-345$, Miarch 1938 .

[6] A. I. Uzkor, "An approach to the problem of optimum directive antenna design," Dokl. Akad. Nauk USSR, vol. 53, pp. 35-38, 1946.

\section{Electromagnetic Pulse Generation by an Impedance Loaded Dipole Antenna}

Abstract-A theoretical study is made for obtaining the pulse radiated from an impedance loaded dipole. Numerical results are presented for an antenna that is slowly charged and suddenly shorted at the terminals. The impedance loadings are chosen as those on an existing antenna located at Sandia Laboratory, Albuquerque, N. Mex.

\section{INTRODUCTION}

The radiation of a particular pulse shape by a dipole antenna usually requires an altogether different voltage pulse driving the antenna. The determination of the required driving voltage pulse may be extremely difficult. However, if the frequency response of the antenna is essentially flat, then the radiated pulse in the equatorial plane $\theta=\pi / 2$ is proportional to the time derivative of the voltage pulse. Such an antenna, an impedance loaded dipole, was recently studied [1]. The formulation yields the current distribution under steady state conditions. The radiated fields also may be determined for steady state conditions using standard techniques. In this paper these radiated fields are superimposed to obtain the appropriate time history of the fields for the specific voltage pulse excitation. To obtain numberical results the impedance loadings are considered to be those on the "long-wire" antenna at Sandia Laboratory, Albuquerque, N. Mex.

\section{Analysis}

\section{Current Distribution}

Consider a dipole antenna of length $2 h$ to extend from $z=-h$ to $z=h$ (see Fig. 1). The antenna is driven at the center by voltage $V_{0}(\omega), \omega$ is the radian frequency, and is symmetrically loaded with

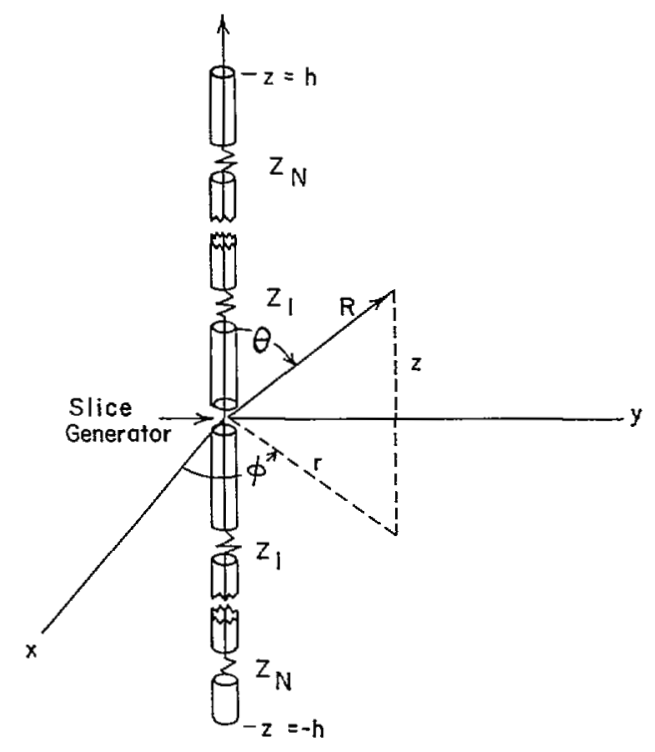

Fig. 1. Impedance-loaded dipole antenna with Cartesian. cylindrical, and spherical coordinates.

impedances $Z_{l}$ at points $\pm z_{l}$ along the antemna axis. For convenience, a slice generator driving mechanism is used. Then the current distribution on the antenna is obtained by solving the integral equation

$$
\begin{aligned}
& \int_{-h}^{h} d z^{\prime} \hat{I}\left(z^{\prime}, \omega\right) K_{a}\left(z-z^{\prime}\right) \\
& -j(4 \pi k / \zeta) \sum_{l=1}^{N} Z_{l} \delta\left(|z|-z_{l}\right) I(z)=-j(4 \pi k / \zeta) \hat{V}_{0}(\omega) \delta(z)
\end{aligned}
$$

where

$$
\begin{aligned}
& K_{a}\left(z-z^{\prime}\right)=\left(\partial^{2} / \partial z^{2}+k^{2}\right) \\
& \cdot\left(\exp \left\{-j k\left[\left(z-z^{\prime}\right)^{2}+a^{2}\right]^{1 / 2}\right\} /\left[\left(z-z^{\prime}\right)^{2}+a^{2}\right]^{1 / 2}\right)
\end{aligned}
$$

and $k=\omega / c$ is the propagation constant and $\zeta \simeq 120 \pi$ ohms is the wave impedance of free space.

The solution to ( 1 ) may be effected by using a finite Fourier series representation for the current distribution. It is

$$
\begin{aligned}
\hat{I}(z, \omega)= & -j \frac{4 \pi \hat{V}_{0}(\omega)}{\zeta} \\
& \cdot\left\{\sum_{M=1}^{M} I_{m} \cos \left[\frac{(2 m+1) \pi}{2} \frac{z}{h}\right]+C \sin k(h-|z|)\right\} .
\end{aligned}
$$

The constant $C$ is chosen to expedite the solution and the expansion coefficients are obtained by solving a system of linear equations resulting from substituting (3) into (1) [1]. To obtain the current distribution on the antenna when driven by a pulsed voltage, $\hat{V}_{0}(\omega)$ is taken as the Fourier transform of the voltage pulse and the resulting $\hat{I}(z, \omega)$ is interpreted as the Fourier transform of the current pulse. Hence the time history of the resulting current is obtained by taking the inverse Fourier transform of $I(z, \omega)$, i.e.,

$$
I(z, t)=\frac{1}{(2 \pi)^{1 / 2}} \int_{-\infty}^{\infty} d \omega \hat{I}(z, \omega) \exp (j \omega t)
$$

If one requires $I(z, t)$ to be real, then $\hat{I}(z,-\omega)=\hat{I}^{*}(z, \omega)$. This yields

$$
I(z, t)=(2 / \pi)^{1 / 2} \int_{0}^{\infty} d \omega \operatorname{Re}\{I(z, \omega) \exp (j \omega t)\}
$$

